\title{
Decreased elastin in vessel walls puts the pressure on
}

\section{Jeanine D'Armiento}

Department of Medicine, Columbia University College of Physicians and Surgeons, New York, New York, USA

Mice haploinsufficient for elastin develop structural changes in vessel walls similar to those seen in patients with mutations in the elastin gene. A new study (see the related article beginning on page 1419) demonstrates that due to mechanical changes in the vessel wall, these animals exhibit increased mean arterial pressures. The results evoke the possibility that alterations in elastin may contribute to the development of essential hypertension in patients.

J. Clin. Invest. 112:1308-1310 (2003). doi:10.1172/JCI200320226.

Investigators have understood that the arrangement of the ECM is critical in the formation of organ structures during development and in the remodeling of tissues after injury. However, the ECM is perceived to play a passive role in dynamic pathological processes such as heart failure and hypertension. This misconception largely results from difficulties in interpreting the pathology seen in human diseased tissue without an understanding of the full phenotypic course of the pathologic process over time. The use of transgenic animal studies now allows us to introduce gain of function and loss of function mutations so as to evaluate the direct role of ECM molecules in disease pathogenesis. There are multiple examples of dominant mutations in structural ECM molecules that lead to abnormal tissue formation and disease phenotypes (1). In this issue of the JCI, Faury and colleagues present an interesting example

Address correspondence to: Jeanine

D'Armiento, Department of Medicine,

Columbia University College of Physicians and Surgeons, Physicians and Surgeons 9-449, 622 West 168th Street, New York, New York 10032, USA. Phone: (212) 305-3745;

Fax: (212) 305-5052;

E-mail: jmd12@columbia.edu.

Conflict of interest: The author has declared that no conflict of interest exists.

Nonstandard abbreviations used:

supravalvular aortic stenosis (SVAS); mean arterial pressure (MAP). of a dominant mutation in an ECM protein that results in developmental structural changes that ultimately cause the animals to acquire hypertension in their adult life (2).

\section{Elastin and vessel formation}

Elastin constitutes $50 \%$ of the dry weight of the aorta. During vessel development, elastin synthesized by smooth muscle cells forms elastic fibers that are arranged into concentric rings of elastic lamellae around the arterial lumen. Each elastic lamella alternates with a ring of smooth muscle, forming a lamellar unit. The elastic lamellae allow an artery to comply with the increased hemodynamic stress of cardiac systole and maintain sufficient blood pressure during diastole (Figure 1) (3).

Variations in the lamellar subunits (defined as the elastic lamellae and adjacent smooth muscle cells) determine the distribution and magnitude of the tensile strength of the vessel. Hence, the greatest number of lamellar units is found in the larger, more proximal vessels that experience higher wall stress, indicating a linear relationship between the number of lamellar units and tensional force within the vessel wall $(4,5)$.

\section{Elastin mutations in disease}

Three clinical conditions have been linked to a mutation or deletion of the elastin gene, ELN. Autosomal dominant cutis laxa, a primarily cutaneous condition, is the result of frameshift mutations in ELN that influence elastic fiber structure through a dominant-negative effect (6). Several years ago, investigators identified mutations in $E L N$ in patients with supravalvular aortic stenosis (SVAS). SVAS is an autosomal dominant disorder caused by intragenic deletions or by a large spectrum of mutations within the elastin gene $(7,8)$. These result in functional haploinsufficiency through either nonsense-mediated decay of mRNA from the mutant allele or the production of a nonfunctional protein $(9,10)$. Narrowing of the ascending aorta is a dominant feature of SVAS, but other arteries, including pulmonary arteries, are often also affected. If not corrected, SVAS may lead to cardiac hypertrophy and heart failure (11). Finally, Williams syndrome, a neurodevelopmental disorder that has SVAS as a component, develops as a consequence of submicroscopic deletions within chromosomal subunit $7 q 11.23$ involving the whole of the ELN gene (12).

\section{Alterations in elastin content change arterial wall structure}

To directly investigate the consequence of elastin mutations on vessel formation, a mouse with a complete loss of function in the Eln gene was generated (13). The elastin-null mice died of obstructive arterial disease due to subendothelial cell proliferation and reorganization of smooth muscle. These changes occurred in isolated organ cultures of arteries and were not subject to hemodynamic stress (13).

The characterization of mice haploinsufficient for elastin $\left(E \ln ^{+/}\right)$ revealed a role for elastin in the formation of vessel wall structure (14). The arteries of $\mathrm{Eln}^{+/-}$mice exhibited thinner elastic lamellae and an increased number of smooth muscle cell layers. Most interestingly, these identical changes have been observed in the arteries of patients with SVAS (14).

In the present report, Faury et al. (2) meticulously examined the mechanical 


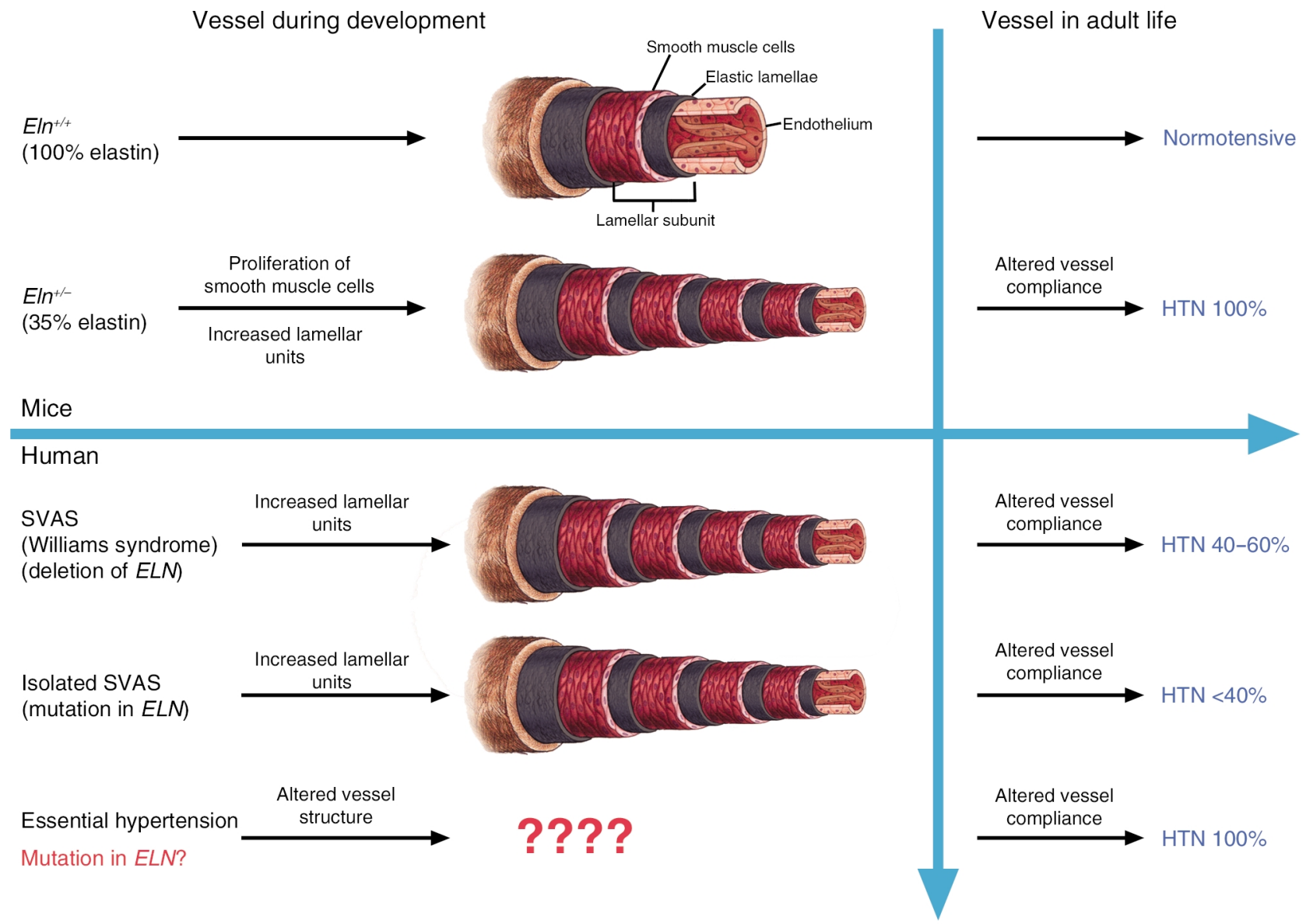

Figure 1

Haploinsufficiency of elastin in mice leads to developmental changes in the vessel wall, resulting in an increase in lamellar units. During the adult life of the animal, these structural changes translate into altered vessel compliance and a significant increase in the mean arterial pressure. These changes are similar to what is observed in two diseases identified as having mutations in the elastin gene; however, the incidence of hypertension (HTN) is lower in the human diseases. These intriguing findings raise the possibility that patients with essential hypertension, who exhibit decreased compliance of their arterial vessels, may have mutations in their elastin gene. Such mutations would lead to structural changes in the vessel during development that predisposes the patients to the development of hypertension later in life.

properties of the arteries of the $E \mathrm{ln}^{+/-}$ mice compared with normal $\mathrm{Eln}^{+/+}$mice, correlating changes in inner and outer vessel diameter with alterations in transmural pressure. The $E \ln ^{+/}$animals were stably hypertensive with mild cardiac hypertrophy and did not exhibit the hypertension-induced arterial wall hypertrophy and decreased distensibility of large elastic arteries associated with essential hypertension (2). The mean arterial pressure (MAP) of $\mathrm{Eln}^{+/-}$mice could be reduced with angiotensin II inhibitors, and renin levels were twofold greater than in $\mathrm{Eln}^{+/+}$mice, suggesting that the renin-angiotensin system plays a role in maintaining the high blood pressure of the $E \ln ^{+/}$mice (2).

The results of this study provide insight into how hemodynamic forces resulting from altered matrix structure influence vascular development (Figure 1). Most importantly, these mutant Eln animals will be extremely useful in identifying the mechanism of hypertension in arteriopathies associated with elastin haploinsufficiency.

\section{Overview and future questions}

Vessels of patients with essential hypertension exhibit decreased arterial compliance and increased vascular resistance with an increase in vascular tone (15). Hypertensive patients maintain the decreased compliance at the same pressures as normotensive patients, implying that functional and/or structural changes other than pressuremediated stretching of arteries contribute toward reducing arterial compliance $(16,17)$. The discovery of an alteration in vessel compliance in the
$E \mathrm{ln}^{+/-}$mice along with increased MAP suggests that vessel elastin in patients with hypertension should be examined. Mutations in the elastin gene could ultimately be a cause of hypertension.

The mechanism by which a change in elastin content leads to alterations in cell signaling and subsequent structural changes in the vessel wall remains to be determined. Matrix molecules bind to integrin receptors, and any alteration in the structural components of the matrix could consequentially alter signaling through the integrin receptors. Additionally, it will be interesting to determine whether elastin loss leads to structural changes in other organs of the $\mathrm{Eln}^{+/}$ mice in response to increased mechanical stress. For example, the lungs from the $\mathrm{Eln}^{+/-}$mice would presum- 
ably have diminished elastin content. Since the rodent lung continues to develop until two months after birth, one wonders whether Eln ${ }^{+/-}$lungs undergo structural reorganization when exposed to postnatal transmural pressure and what the consequences of such reorganization might be on pulmonary function.

1. Helminen, H.J., Saamanen, A.M., Salminen, H., and Hyttinen, M.M. 2002. Transgenic mouse models for studying the role of cartilage macromolecules in osteoarthritis. Rheumatology. 41:848-856.

2. Faury, G., et al. 2003. Developmental adaptation of the mouse cardiovascular system to elastin haploinsufficiency. J. Clin. Invest. 112:1419-1428. doi:10.1172/JCI200319028.

3. Parks, W.C., Pierce, R.A., Lee, K.A., and Mecham, R.P. 1993. Elastin. Advances in Molecular and Cellular Biology. 6:133-182.
4. Leung, D.Y.M., Glagov, S., and Mathews, M.B 1977. Elastin and collagen accumulation in rabbit ascending aorta and pulmonary trunk during postnatal growth: correlation of cellular synthetic response with medial tension. Circ. Res. 41:316-323.

5. Clark, J.M., and Glagov., S. 1985. Transmural organization of the arterial media. The lamellar unit revisited. Arteriosclerosis. 5:19-34.

6. Tassabehji, M., et al. 1998. An elastin gene mutation producing abnormal tropoelastin and abnormal elastic fibres in a patient with autosomal dominant cutis laxa. Hum. Mol. Genet. 7:1021-1028.

7. Curran, M.E., et al. 1993. The elastin gene is disrupted by a translocation associated with supravalvular aortic stenosis. Cell. 73:159-168.

8. Ewart, A.K., Jin, W.S., Atkinson, D., Morris, C.A., and Keating, M.T. 1994. Supravalvular aortic stenosis associated with a deletion disrupting the elastin gene. J. Clin. Invest. 93:1071-1077.

9. Urban, Z., et al. 1999. Supravalvular aortic stenosis: a splice site mutation within the elastin gene results in reduced expression of two aberrantly spliced transcripts. Hum. Genet. 104:135-142. 10. Urban, Z., et al. 2000. Isolated supravalvular aor- tic stenosis; functional haploinsufficiency of the elastin gene as a result of nonsense-mediated decay. Hum. Genet. 106:577-588.

11. Chowdhury, T., and Reardon, W. 1999. Elastin mutation and cardiac disease. Pediatr. Cardiol. 20:103-107.

12. Lowery, M.C., Morris, C.A., and Ewart, A.K. 1995 Strong correlation of elastin deletions, detected by FISH, with Williams Syndrome: evaluation of 235 patients. Am. J. Hum. Genet. 57:49-53.

13. Li, D.Y., et al. 1998. Elastin is an essential determinant of arterial morphogenesis. Nature. 393:276-280.

14. Li, D., et al. 1998. Novel arterial pathology in mice and humans hemizygous for elastin. J. Clin. Invest. 102:1783-1787.

15. Ting, C.T., et al. 1986. Arterial hemodynamics in human hypertension. J. Clin. Invest. 78:1462-1471.

16. Simon, A.C., Levenson, J., Chau, N.P., and PithoisMerli, I. 1992. Role of arterial compliance in the physiopharmacological approach to human hypertension. J. Cardiovasc. Pharmacol. 5(Suppl.):S11-S20.

17. Armentano, R., et al. 1991. Mechanical pressure versus intrinsic effects of hypertension on large arteries in humans. Hypertension. 18:657-664.

\section{The origin of FOXP3-expressing CD4 ${ }^{+}$ regulatory $T$ cells: thymus or periphery}

\section{Shimon Sakaguchi}

Department of Experimental Pathology, Institute for Frontier Medical Sciences, Kyoto University, Kyoto, Japan

Laboratory for Immunopathology, RIKEN Research Center for Allergy and Immunology, Yokohama, Japan

Naturally arising $\mathrm{CD}^{+}$regulatory $\mathrm{T}$ cells, which engage in the maintenance of immunologic self-tolerance, specifically express FOXP3, which encodes a transcription-repressor protein. Genetic defects in FOXP3 cause IPEX, an X-linked autoimmune/inflammatory syndrome. With FOXP3 as a specific marker for regulatory $\mathrm{CD}^{+} \mathrm{T}$ cells in humans, it is now possible to determine their origin and developmental pathway (see the related article beginning on page 1437).

J. Clin. Invest. 112:1310-1312 (2003). doi:10.1172/JCI200320274.

The immune system discriminates between self and non-self, maintaining immunologic self-tolerance (i.e., unresponsiveness to self-constituents). It is known that potentially hazardous selfreactive $\mathrm{T}$ and $\mathrm{B}$ cells are clonally delet-

Address correspondence to: Shimon Sakaguchi, Department of Experimental Pathology, Institute for Frontier Medical Sciences, Kyoto University, 53 Shogin

Kawahara-cho, Sakyo-ku, Kyoto 606-8507, Japan. Phone: 81-75-751-3888;

Fax: 81-75-751-3820;

E-mail: shimon@frontier.kyoto-u.ac.jp.

Conflict of interest: The author has declared that no conflict of interest exists.

Nonstandard abbreviations used: regulatory

$\mathrm{T}\left(\mathrm{T}_{\mathrm{R}}\right)$; inflammatory bowel disease (IBD);

$\mathrm{T}$ cell receptor (TCR). ed at immature stages of their development or inactivated upon encounter with self-antigens in the periphery. There is now accumulating evidence that, in addition to these passive mechanisms of self-tolerance, a population of $\mathrm{CD}^{+} \mathrm{T}$ cells, called regulatory $\mathrm{T}$ cells $\left(T_{R}\right.$ cells), engage in the maintenance of peripheral self-tolerance by actively suppressing the activation and expansion of self-reactive T cells (1-3). The majority, if not all, of such naturally occurring $\mathrm{CD}^{+} \mathrm{T}_{\mathrm{R}}$ cells constitutively express CD25 (IL-2 receptor $\alpha$ chain) in the physiologic state. Indeed, removal of $\mathrm{CD}^{2} 5^{+} \mathrm{CD} 4^{+} \mathrm{T}$ cells, which constitute $5-10 \%$ of $\mathrm{CD}^{+} \mathrm{T}$ cells in rodents and humans, leads to sponta- neous development of various autoimmune diseases in otherwise normal mice (4). The removal of $\mathrm{CD} 25^{+} \mathrm{CD} 4^{+}$ $\mathrm{T}_{\mathrm{R}}$ cells also triggers excessive or misdirected immune responses to microbial antigens, causing immunopathology, such as inflammatory bowel disease (IBD), due to hyper-reaction of the remaining $\mathrm{T}$ cells to commensal bacteria in the intestine (3).

\section{FOXP3: master control gene for the development and function of natural $\mathrm{CD4}^{+} \mathrm{T}_{\mathrm{R}}$ cells}

There is now evidence not only for the presence of $\mathrm{CD} 25^{+} \mathrm{CD} 4^{+} \mathrm{T}_{\mathrm{R}}$ cells in humans but also for their essential roles in controlling autoimmunity, immunopathology, and allergy in human diseases (5). This is best illustrated by IPEX (immune dysregulation, polyendocrinopathy, enteropathy, X-linked syndrome), a rare monogenic disease of male children that is accompanied by autoimmune disease (such as type 1 diabetes), IBD, and severe allergy similar to those produced in mice by depletion of $\mathrm{CD} 25^{+} \mathrm{CD} 4^{+} \mathrm{T}_{\mathrm{R}}$ cells (6). The causative gene, FOXP3 (Foxp3 in mice), which encodes a transcription repressor (7-10), is specifically expressed in $\mathrm{CD} 25^{+} \mathrm{CD} 4^{+} \mathrm{T}$ cells in the thymus and periphery (11-13). Forced expression of the Foxp3 gene can convert murine naive $T$ cells to $T_{R}$ cells that phenotypically and functionally resemble naturally arising $\mathrm{CD} 25^{+} \mathrm{CD} 4^{+} \mathrm{T}_{\mathrm{R}}$ cells 